\title{
NOVAS TECNOLOGIAS EDUCACIONAIS NO ENSINO DA AUDIOLOGIA
}

\section{New educational technologies in audiology teaching}

\author{
Wanderléia Quinhoneiro Blasca ${ }^{(1)}$, Luciana Paula Maximino (2), \\ Debora Godoy Galdino ${ }^{(3)}$, Karis de Campos ${ }^{(4)}$, Mirela Machado Picolini (5)
}

\section{RESUMO}

Objetivo: elaborar, aplicar e avaliar um modelo de Teleducação Interativa para o ensino da Audiologia, disciplina de Aparelhos de Amplificação Sonora Individual. Métodos: participaram deste estudo 13 alunos do $2^{\circ}$ ano do curso de Fonoaudiologia de uma universidade pública do município de Bauru-SP. O programa de Teleducação Interativa foi elaborado, utilizando um tutor eletrônico, o Cybertutor. Os participantes receberam uma senha de acesso ao conteúdo teórico da disciplina. O prazo estabelecido foi de 30 dias, sendo que o aluno poderia acessar quantas vezes julgasse necessário. Para a avaliação do programa foi realizada a aplicação de dois questionários, sendo um referente ao conteúdo didático-teórico da disciplina de Aparelhos de Amplificação Sonora Individual e outro referente ao objeto de aprendizagem utilizado (Cybertutor). O questionário didático-teórico avaliou a efetividade do programa na aprendizagem do aluno e foi aplicado em dois momentos distintos, ou seja, pré e pós-teste. O questionário referente ao Cybertutor abordou aspectos de aceitação, viabilidade e interatividade. Para a análise dos resultados foi utilizado o teste Wilcoxon $(p \leq 0,05)$ e dados percentuais. Resultados: a diferença estatisticamente significante encontrada na comparação pré e pós-teste com $\mathrm{p}=0,016$, demonstra que o Cybertutor proporcionou o aprendizado do aluno de graduação na área da Audiologia, disciplina de Aparelho de Amplificação Sonora Individual. Quanto à avaliação do Cybertutor, verificou-se que $100 \%$ dos alunos consideram-no explicativo, e de fácil entendimento. Conclusão: o programa de Teleducação Interativa mostrou-se efetivo como material educacional no processo ensino-aprendizagem da Audiologia.

DESCRITORES: Educação a Distância; Tecnologia Educacional; Instrução por Computador; Audiologia

(1) Fonoaudióloga; Professora do Departamento de Fonoaudiologia da Faculdade de Odontologia de Bauru da Universidade de São Paulo, FOB-USP, Bauru, SP, Brasil; Doutora em Distúrbios da Comunicação pelo Hospital de Reabilitação de Anomalias Craniofaciais.

(2) Fonoaudióloga; Professora do Departamento de Fonoaudiologia da Faculdade de Odontologia de Bauru da Universidade de São Paulo, FOB-USP, Bauru, SP, Brasil; Doutora em Ciências Biológicas em Genética Médica e Humana pelo Instituto de Biociências da Universidade Estadual Paulista.

(3) Fonoaudióloga; Responsável técnica da Secretaria Municipal de Educação da Estância Turística de Presidente Epitácio, SP, Brasil; Mestranda em Saúde, Interdisciplinaridade e Reabilitação na Faculdade de Ciências Médicas da Universidade Estadual de Campinas.

(4) Fonoaudióloga; Aluna de Pós-Graduação da Faculdade de Odontologia de Bauru da Universidade de São Paulo, FOBUSP, Bauru, SP, Brasil; Mestranda em Fonoaudiologia na Faculdade de Odontologia de Bauru da Universidade de São Paulo.

\section{INTRODUÇÃO}

Com a expansão da Internet, as áreas da informática e da telecomunicação proporcionaram mudanças significativas nos diversos setores que regem o desenvolvimento do país. Desta forma, o setor educacional vem passando nos últimos anos, por um processo de modernização impulsionado pelos avanços tecnológicos.

As interações na teia telemática propiciadas pela Internet tornaram-se um importante instrumento de

(5) Fonoaudióloga; Aluna de Pós-Graduação da Faculdade de Odontologia de Bauru da Universidade de São Paulo, FOBUSP, Bauru, SP, Brasil; Mestranda em Fonoaudiologia na Faculdade de Odontologia de Bauru da Universidade de São Paulo.

Conflito de interesses: inexistente 
difusão do conhecimento ${ }^{1}$, abrindo as fronteiras de salas de aula convencionais desencadeando processos de aprendizagem diferenciados dos até então instituídos ao longo da história ${ }^{2}$. Nesta perspectiva, a Educação a Distância $(\mathrm{EaD})$ é uma modalidade educacional na qual a mediação didático-pedagógica, nos processos de ensino e aprendizagem, ocorre com a utilização de meios e Tecnologias de Informação e Comunicação (TICs).

No art. 80 da atual Lei de Diretrizes e Bases da Educação Nacional, Lei oㅜ 9.394/1996, está preconizado que o poder público incentivará o desenvolvimento e a veiculação de programas de ensino à distância, em todos os níveis e modalidades de ensino e de educação continuada. No Brasil, a educação a distância está em uma fase promissora, pois se tornou uma opção de aquisição de novos conhecimentos, com a finalidade de agregar um maior número de pessoas, diminuir as dificuldades geográficas, minimizar os custos e otimizar 0 tempo ${ }^{3,4}$.

Com relação à formação do Fonoaudiólogo no Brasil, atualmente existem 91 cursos de Graduação em Fonoaudiologia no Brasil ${ }^{5}$, sendo 8 programas de mestrado e 4 programas de doutorado reconhecidos pelo Ministério da Educação ${ }^{6}$. No entanto, a maioria dos cursos de graduação e pós-graduação concentra-se na região Sul e Sudeste.

Tendo em vista o contexto do país, tanto do ponto de vista da extensão territorial bem como, da distribuição heterogênea dos cursos de graduação e pós-graduação em Fonoaudiologia, está evidente a necessidade da elaboração de materiais educacionais que auxiliem no exercício da docência, pensando principalmente na formação e atualização destes futuros profissionais.

Uma alternativa para este cenário educacional é a teleducação, uma vez que esta ferramenta pode atingir grandes contingentes de alunos e profissionais de forma efetiva e sem prejudicar a qualidade do ensino ${ }^{7}$.

Vários estudos estão sendo realizados com 0 intuito de verificar a percepção, aceitação e satisfação dos estudantes de graduação em relação à teleducação ${ }^{8,9}$ bem como a efetividade do ensino/ aprendizagem ${ }^{10}$.

Em relação à Fonoaudiologia, ressaltando a área de Audiologia, alguns estudos ${ }^{11-13}$ desenvolveram materiais educacionais predominantemente no formato de CD-ROM enfatizando o ensino a distância e a aprendizagem do aluno.

Com a criação do Departamento de Teleaudiologia em 2004, no XIX Encontro Internacional de Audiologia, estão sendo desenvolvidos cursos de Audiologia à Distância, bem como materiais educativos para alunos, pacientes e profissionais da área da saúde. Dentre eles pode-se citar o Cybertutor em Audiologia, Homem Virtual sobre o Aparelho de Amplificação Sonora Individual, Portal dos Bebês (website) e o Cyberambulatório em Audiologia, focando a segunda opinião formativa e a discussão de casos clínicos ${ }^{14-16}$.

Estes programas educacionais, mais especificamente o Cybertutor - tutor eletrônico baseado na internet - além de utilizar a metodologia do aprendizado baseado em problemas (PBL) apresenta também recursos de interatividade. Dessa forma, o Cybertutor está sendo utilizado não apenas pelos programas da $\mathrm{EaD}$, mas também para complementar o aprendizado em salas de aulas tradicionais ${ }^{16}$.

Estas práticas educacionais à distância associadas aos recursos interativos possibilitaram o advento da Teleducação Interativa. Dessa forma a Teleducação Interativa é mais do que o simples ato de reunir informações e tecnologias, é a união criteriosa dos recursos de informática e telecomunicação baseados em modelos educacionais, estimulando a interatividade e mantendo o interesse do aluno através de meios de comunicação eficientes e dirigidos ${ }^{17}$.

Sendo assim, este estudo teve como objetivos elaborar, aplicar e avaliar um modelo de Teleducação Interativa baseado na web para o ensino da Audiologia, disciplina de Aparelhos de Amplificação Sonora Individual.

\section{MÉTODOS}

Este estudo foi desenvolvido pelo Departamento de Fonoaudiologia da Faculdade de Odontologia de Bauru da Universidade de São Paulo em parceria com a Disciplina de Telemedicina da Faculdade de Medicina da Universidade de São Paulo, como parte da proposta do projeto contemplado pelo Edital Instituto do Milênio em 2005 - CNPq - "Estação Digital Medica: estratégia de implementação e ampliação da Telemedicina no Brasil".

Para compor a casuística, os alunos do $2^{\circ}$ ano do Curso de Fonoaudiologia de uma universidade pública do município de Bauru-SP, foram convidados a participar voluntariamente deste estudo. Após leitura e a assinatura do Termo de Consentimento Livre e Esclarecido, aqueles que demonstraram interesse foram selecionados. Optou-se pela seleção dos alunos que cursavam o $2^{\circ}$ ano, visto que os mesmos já estavam cursando as disciplinas de audiologia. Participaram deste estudo 13 indivíduos, do sexo feminino da faixa etária entre 18 e 20 anos.

O modelo do programa de Teleducação Interativa foi elaborado, utilizando-se de um tutor 
eletrônico, o Cybertutor, que como objeto de aprendizagem tornou-se possível:

- Fragmentar o curso em 4 módulos facilitando o aprendizado do aluno, pois o mesmo poderia realizar uma avaliação de reforço no final de cada módulo. Desta forma, o aluno avaliava seu conhecimento teórico e realizaria nova leitura caso fosse necessário.

- Utilizar material ilustrativo através das iconografias em 3D do Projeto Homem Virtual da Audição.

- Utilizar o simulador de caso: a avaliação do conhecimento é baseada em decisões a serem feitas a partir de um caso clínico, estimulando o raciocínio teórico prático do aluno através dos desafios.

- Realizar lista de discussões via internet, permitindo maior interação entre os alunos.

O conteúdo programático do Cybertutor foi dividido em 4 módulos, respeitando a base teórica dos principais tópicos da Disciplina de Amplificação Sonora Individual, a saber:

- Módulo I: Componentes básicos e processamento do sinal.

- Módulo II: Características físicas e eletroacústicas.

- Módulo III: Princípios de pré-seleção do ApareIho de Amplificação Sonora Individual.

- Módulo IV: Moldes auriculares.

Os alunos participantes receberam uma senha de acesso ao Cybertutor, disponibilizando o conteúdo teórico da Disciplina de Aparelho de Amplificação Sonora Individual. O prazo de acesso estabelecido foi de 30 dias, sendo que o aluno poderia acessar quantas vezes julgasse necessário. Nesse processo, o aluno direciona seu próprio aprendizado, mas sempre sendo conduzido pelo Cybertutor que verifica seu desempenho e sua programação de estudo.

O processo de avaliação do programa de Teleducação Interativa foi realizado presencialmente, utilizando-se de uma avaliação escrita.

Foi realizada a aplicação de 2 questionários avaliativos, sendo um referente ao material didáticoteórico da Disciplina de Aparelho de Amplificação Sonora Individual e outro referente ao objeto de aprendizagem utilizado (Cybertutor).

O questionário didático-teórico foi elaborado para avaliar a efetividade do programa de Teleducação na aprendizagem do aluno. Dessa forma, foi aplicado em dois momentos distintos, ou seja, pré e pós-teste. A avaliação pré-teste foi realizada antes do acesso ao conteúdo teórico do Cybertutor, e a avaliação pós-teste foi realizada após o período de
30 dias de acesso. Este questionário foi composto por 48 questões de múltipla escolha, com apenas 1 alternativa correta.

O questionário utilizado para avaliar o Cybertutor, como objeto de aprendizagem englobou aspectos de aceitação, viabilidade e interatividade. Este questionário foi composto por 10 questões, com 2 opções de resposta (sim e não).

A aprovação do Comitê de Ética em Pesquisa da Faculdade de Odontologia de Bauru está protocolada sob parecer $n^{\circ}$ 115/2006.

Para a análise dos resultados, o questionário didático-teórico foi comparado nas situações pré e pós-teste. Seguindo o objetivo, para verificar a efetividade do programa de ensino proposto, foi utilizado o teste de Wilcoxon adotando $5 \%(p \leq 0,05)$ o valor para rejeição da hipótese de nulidade $(\mathrm{Ho})$. O questionário para avaliar o objeto de aprendizagem (Cybertutor) foi descrito de forma percentual.

\section{RESULTADOS}

As Figuras 1, 2 e 3 ilustram o material do programa de Teleducação Interativa, baseado no Cybertutor.

A Figura 1 mostra a fragmentação do curso teórico em módulos, exemplificando o Módulo I sobre os componentes básicos e processamento do sinal.

A Figura 2 mostra o simulador de caso, com casos clínicos e desafios, estimulando o raciocínio teórico do aluno.

A Figura 3 mostra a interatividade entre os participantes através da lista de discussão.

O desempenho dos alunos em relação ao conteúdo didático-teórico do programa de Teleducação Interativa encontra-se descrito na Tabela $1 \mathrm{em}$ número de acertos e porcentagem.

Os resultados demonstraram haver diferença estatisticamente significante quanto à comparação do desempenho dos alunos na situação pré e pósteste, pois os alunos apresentaram melhor desempenho na avaliação pós-teste (média= $63,3 \%$ ) em relação à pré-teste (média $=37,1 \%$ ), com $p=0,016$.

No que se refere à análise dos dados por módulo do programa de Teleducação Interativa, conclui-se de acordo com os dados da Tabela 2 que os alunos apresentaram melhor desempenho em todos os módulos na avaliação pós-teste.

A Tabela 3 apresenta os dados do questionário de avaliação do Cybertutor, quanto objeto de aprendizagem utilizado no programa de Teleducação Interativa. 


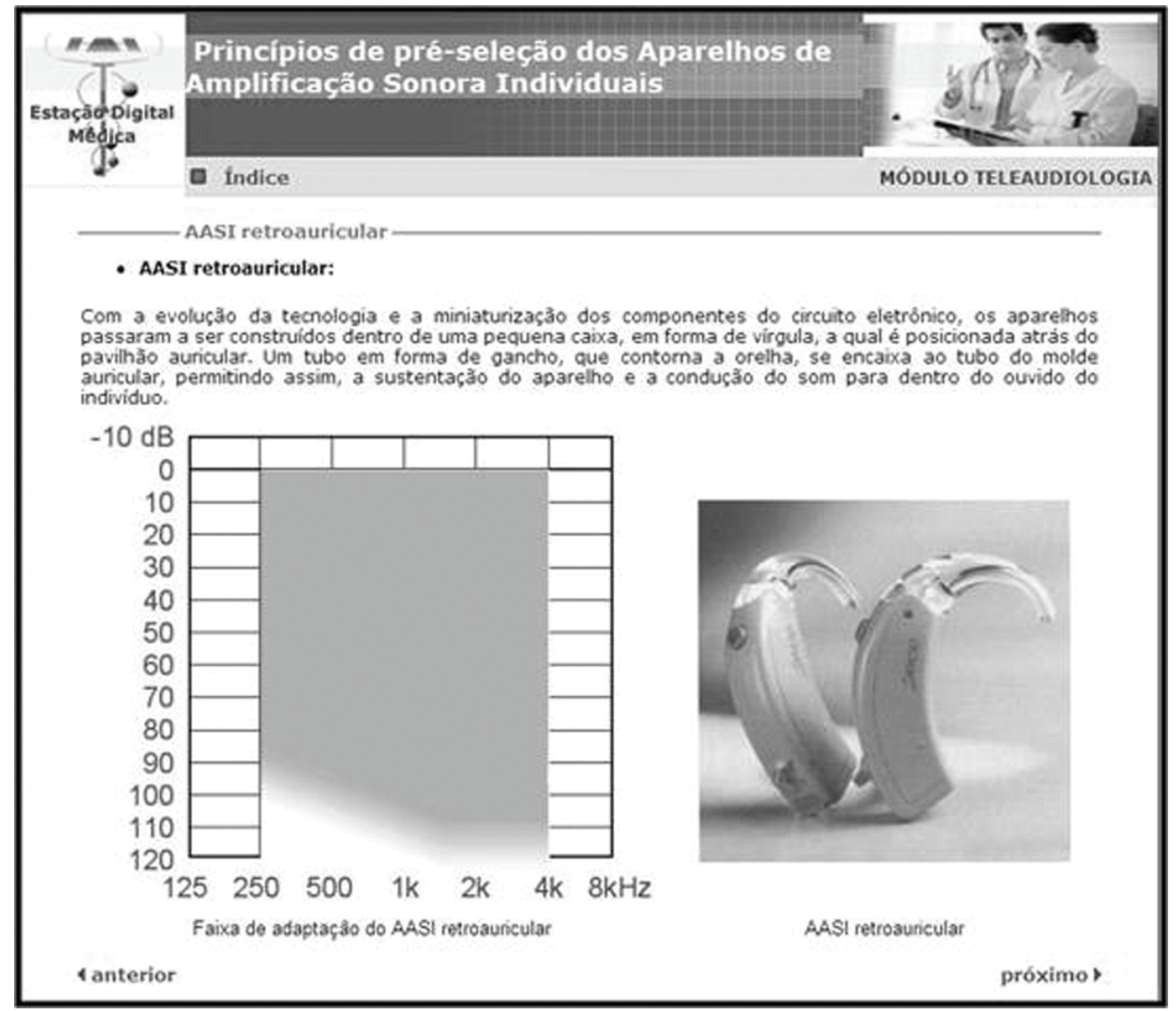

Figura 1 - Tela do Cybertutor (Módulo I)

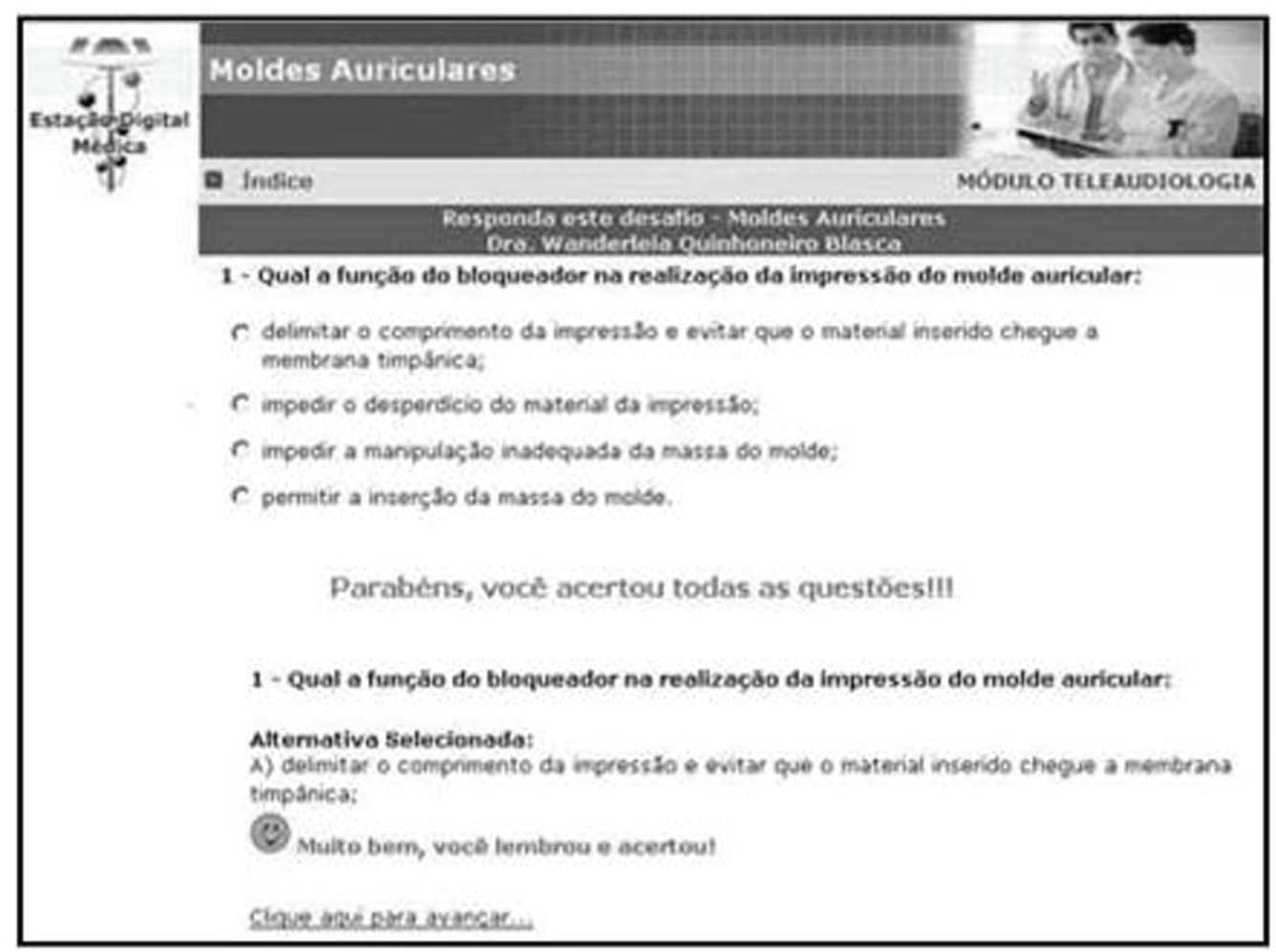

Figura 2 - Tela do Cybertutor (Simulador de caso) 


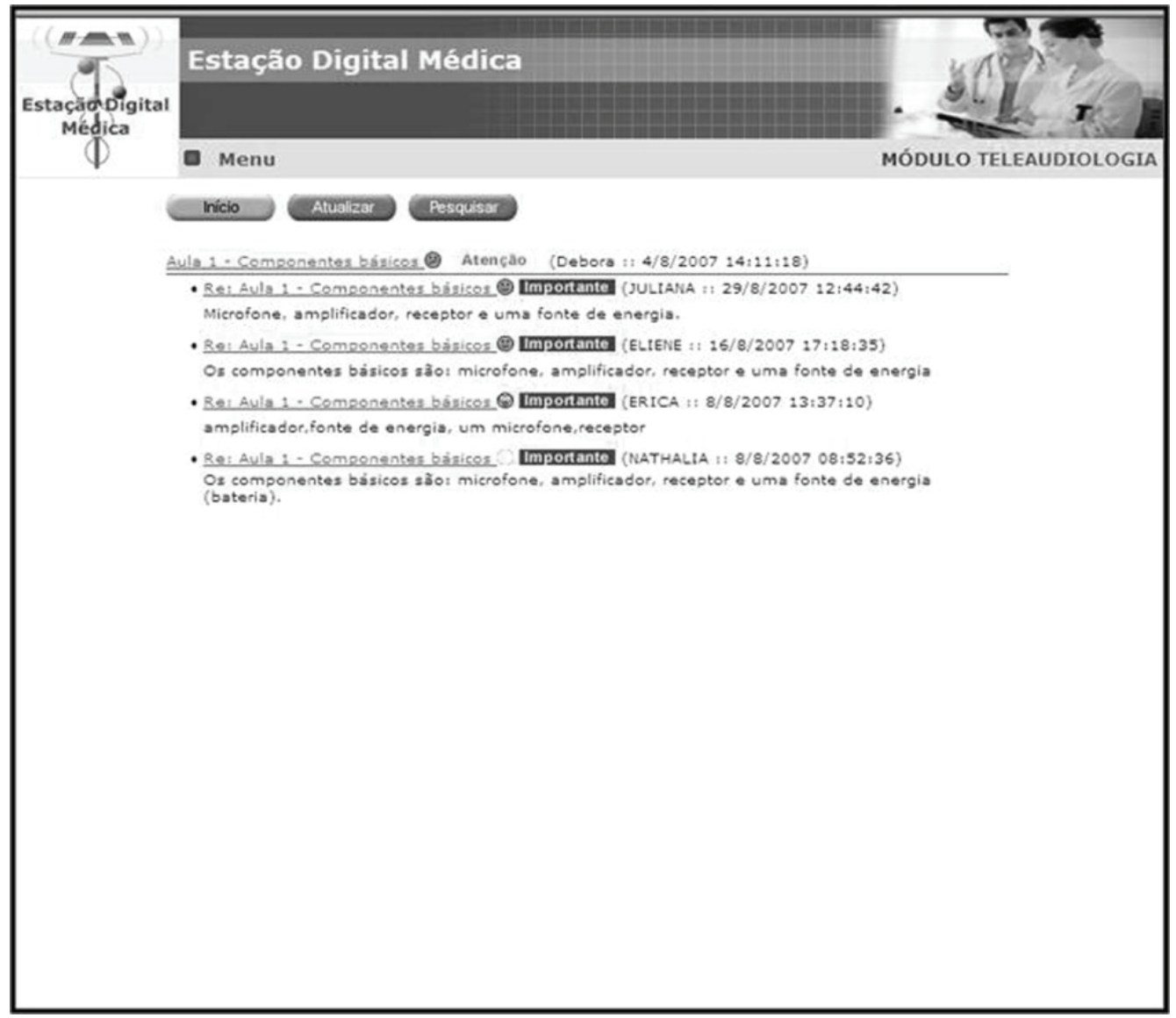

Figura 3 - Tela do Cybertutor (Lista de Discussão)

Tabela 1 - Desempenho dos alunos na avaliação pré e pós-teste

\begin{tabular}{ccccc}
\hline \multirow{2}{*}{ Alunos } & \multicolumn{2}{c}{ Acertos pré-teste } & \multicolumn{2}{c}{ Acertos pós-teste } \\
\cline { 2 - 5 } & $\mathbf{N}$ & $\mathbf{( \% )}$ & $\mathbf{N}$ & $\mathbf{( \% )}$ \\
\hline $\mathbf{1}$ & 25 & $52,1 \%$ & 19 & $39,6 \%$ \\
$\mathbf{2}$ & 17 & $35,4 \%$ & 43 & $89,6 \%$ \\
$\mathbf{3}$ & 18 & $37,5 \%$ & 25 & $52,1 \%$ \\
$\mathbf{4}$ & 24 & $50,0 \%$ & 31 & $64,6 \%$ \\
$\mathbf{5}$ & 14 & $29,2 \%$ & 26 & $54,2 \%$ \\
$\mathbf{6}$ & 24 & $50,0 \%$ & 24 & $50,0 \%$ \\
$\mathbf{7}$ & 24 & $50,0 \%$ & 32 & $66,7 \%$ \\
$\mathbf{8}$ & 24 & $50,0 \%$ & 11 & $22,9 \%$ \\
$\mathbf{9}$ & 14 & $29,2 \%$ & 33 & $68,8 \%$ \\
$\mathbf{1 0}$ & 21 & $43,8 \%$ & 42 & $87,5 \%$ \\
$\mathbf{1 1}$ & 17 & $35,4 \%$ & 27 & $56,2 \%$ \\
$\mathbf{1 2}$ & 16 & $33,3 \%$ & 43 & $89,6 \%$ \\
$\mathbf{1 3}$ & 26 & $54,2 \%$ & 39 & $81,2 \%$ \\
\hline Média de acertos & 20,3 & $37,1 \%$ & 30,4 & $63,3 \%$ \\
\hline
\end{tabular}


Tabela 2 - Porcentagem de acertos por módulo do programa de Teleducação Interativa

\begin{tabular}{ccccc}
\hline & $\begin{array}{c}\text { Módulo I } \\
\text { (\%) }\end{array}$ & $\begin{array}{c}\text { Módulo II } \\
\mathbf{( \% )}\end{array}$ & $\begin{array}{c}\text { Módulo III } \\
\mathbf{( \% )}\end{array}$ & $\begin{array}{c}\text { Módulo IV } \\
\text { (\%) }\end{array}$ \\
\hline Acertos pré-teste & $34,6 \%$ & $36,9 \%$ & $56,9 \%$ & $38,5 \%$ \\
Acertos pós-teste & $68,3 \%$ & $48,5 \%$ & $70,8 \%$ & $65,1 \%$ \\
\hline
\end{tabular}

Tabela 3 - Questionário de avaliação do Cybertutor

\begin{tabular}{lcc}
\hline Questões & Sim (\%) & Não (\%) \\
\hline Você achou que o Cybertutor é um recurso didático que propicia & $100 \%$ & - \\
maior entendimento ao aluno? & & \\
Você achou o Cybertutor explicativo e de fácil entendimento? & $100 \%$ & $23 \%$ \\
Você achou o Cybertutor interativo? & $77 \%$ & $77 \%$ \\
Você mudaria as páginas (“layouf')? & $23 \%$ & $85 \%$ \\
Você mudaria o sistema de navegação ("links")? & $15 \%$ & $85 \%$ \\
Você mudaria as imagens? & $15 \%$ & $85 \%$ \\
Você mudaria a forma de apresentação dos textos? & $15 \%$ & \\
\hline
\end{tabular}

\section{DISCUSSÃO}

Um dos principais avanços do século XXI é o reconhecimento da Educação como um dos fatores de maior importância para o desenvolvimento humano. No panorama internacional, a diferença básica entre as nações pobres que estão "emergindo" e as que estão estagnadas consiste no grau em que se dedicaram a formar e ampliar seus programas de Educação ${ }^{17}$.

Nos dias de hoje, possibilitar o acesso à EaD, com os mais diferentes meios de comunicação torna-se fundamental e ao mesmo tempo desafiador. Em todo esse processo, diversas barreiras de comunicação podem ser identificadas, sendo a primeira relacionada ao conteúdo didático-teórico, mais especificamente ao tipo de linguagem utilizada, à forma de apresentação da informação, ao material didático utilizado, à facilidade do acesso ao material e finalmente, a avaliação da aprendizagem.

Assim, esse estudo teve como proposta elaborar um programa de Teleducação Interativa na área de audiologia, disciplina de Aparelho de Amplificação Sonora Individual. A partir desse trabalho poderão ser direcionadas novas propostas de ensino e aprendizado em Audiologia, favorecendo profissionais e pacientes que vivem em regiões mais distantes.

De acordo com os dados descritos na Tabela 1 , verificou-se que a média da porcentagem total dos acertos na avaliação pré-teste foi de $37,1 \%$ e na avaliação pós-teste foi de $63,3 \%$. No entanto, $3(23 \%)$ participantes apresentaram resultado insatisfatório na avaliação pós-teste, na qual o aluno 1 apresentou $52,1 \%$ na avaliação pré-teste e 39,6\% na avaliação pós-teste, o aluno 8 apresentou $50 \%$ na avaliação pré-teste e $22,9 \%$ na avaliação pósteste e o aluno 6 apresentou o mesmo resultado tanto na avaliação pré como na avaliação pós-teste $(50 \%)$. Estes resultados podem estar relacionados ao próprio participante, pois a motivação, a disponibilidade, a expectativa, a satisfação e principalmente o interesse são fatores interferentes desta modalidade educacional, onde o aluno direciona seu aprendizado ${ }^{18,19}$.

A diferença estatisticamente significante encontrada na comparação pré e pós-teste com $p=0,016$, demonstra que o objeto de aprendizagem (Cybertutor) elaborado proporcionou o aprendizado do aluno de graduação na área da Audiologia, disciplina de Aparelho de Amplificação Sonora Individual.

Resultados semelhantes foram evidenciados na Tabela 2, enfatizando a melhora dos resultados pósteste em todos os módulos do programa de Teleducação Interativa. Estes dados corroboram a literatura consultada ${ }^{11-13,16}$ evidenciando a efetividade de materiais educacionais voltados para o ensino da Audiologia na relação ensino-aprendizagem.

Analisando os dados da Tabela 3 referentes à avaliação do objeto de aprendizagem (Cybertutor), verificou-se que $100 \%$ dos alunos afirmam que o Cybertutor é explicativo e proporciona 
maior possibilidade de entendimento. Quando foi sugerido se o aluno gostaria de fazer alguma modificação no Cybertutor, somente 15\% dos alunos relataram modificar as páginas (layout), o sistema de navegação (links) e as imagens. Em relação à interatividade, $73 \%$ dos alunos afirmam que o Cybertutor é um recurso interativo.

O Cybertutor enquanto objeto de aprendizado interativo utilizado na educação a distancia demonstra ser uma excelente opção para a aquisição do conhecimento ${ }^{16}$. Outros estudos ${ }^{3,20,21}$ também utilizaram o Cybertutor, verificando grande aceitação e efetividade do aprendizado, além de estimular a interatividade com as listas e fóruns de discussões.

Nesta perspectiva, educar não é fornecer grandes quantidades de informações, mas desenvolver uma estratégia lógica e coerente, em linguagem compatível ao nível dos estudantes, associada a materiais interativos que facilitem a memorização e aprendizado dos alunos ${ }^{17}$.

\section{CONCLUSÃO}

Diante da progressão do número de acertos e da diferença estatisticamente significante entre as avaliações pré e pós-teste, o programa de Teleducação Interativa mostrou-se efetivo como material educacional no processo ensino-aprendizagem da Audiologia, disciplina de Aparelho de Amplificação Sonora Individual.

Cabe ressaltar que outros estudos devam ser realizados, visando aprimorar e ampliar a Educação a Distância na Fonoaudiologia.

\section{AGRADECIMENTOS}

Agradecemos à Disciplina de Telemedicina da Faculdade de Medicina da Universidade de São Paulo (FMUSP), em especial ao Prof. Dr. Chao Lung Wen pela parceria na realização deste estudo.

\begin{abstract}
Purpose: to develop, implement and evaluate a model of Interactive Tele-education for teaching Audiology, discipline of hearing aids. Methods: 13 students from 2nd year of speech-language pathology in a public university of the city of Bauru-SP. The program of Interactive Tele-education was prepared, using a 'Cybertutor' (a website-based educational environment). The students received a password to access the theoretical content of the discipline. The deadline was 30 days, the students could access as often as they deemed being necessary. For evaluating the application, we applied 2 questionnaires, one related to the didactic-theoretical content of the discipline of hearing aids and the other one related to the used learning object (Cybertutor). The questionnaire assessed the didactictheoretical effectiveness of the program of the student in learning and was applied at two different times, pre-testing and post-testing. The questionnaire addressed issues related to Cybertutor acceptance, feasibility and interactivity. For analyzing the results we used the Wilcoxon test $(p<0.05)$ and percentage data. Results: statistically significant differences were found when comparing pre-testing and posttesting with $p=0.016$, showing that the Cybertutor provided for the learning of students in the area of Audiology, the discipline of hearing aids. As for the evaluation of Cybertutor, it was found that $100 \%$ of students considered it explanatory, and easy to understand. Conclusion: Interactive Tele-education of the program was effective as an educational material in Audiology teaching/learning process.
\end{abstract}

KEYWORDS: Education, Distance; Educational Technology; Computer-Assisted Instruction; Audiology

\section{REFERÊNCIAS}

1. Preger CM. Educação médica continuada a distância em endocrinologia e metabologia. Arq Bras Endocrinol Metabol. 2005; 49(4):584-95.

2. Mendes TS. Intervenções educativas em ambientes virtuais: investigando possibilidades metodológicas. Educ Bras. 2007; 29(58/59): $101-21$
3. Corrêa FG. Treinamento de profissionais de educação física para o reconhecimento precoce de doenças osteoarticulares através do uso de teleducação interativa [dissertação]. São Paulo (SP): Faculdade de Medicina da Universidade de São Paulo; 2008.

4.Spinardi ACP, Blasca WQ, Maximino LP. Genética e fonoaudiologia: aprendizado baseado na teleducação. Pró-Fono. 2009; 20(Supl):42-4. 
5. Conselho Federal de Fonoaudiologia [homepage na internet]. Cursos de Fonoaudiologia no Brasil. Brasília: 2009. [acesso em 27 de agosto 2009]. Disponível em: http://www.fonoaudiologia.org.br// servlet/ConsultaNoticia? acao $=\mathrm{V} \&$ notld $=56$

6. Brasil. Ministério da Educação [homepage na internet]. Distribuição de programas de pósgraduação no Brasil. Brasília: 2009. [acesso em 27 de agosto 2009]. Disponível em: http://geocapes. capes.gov.br

7. Spinardi ACP, Blasca WQ, Wen CL, Maximino LP. Telefonoaudiologia: ciência e tecnologia em saúde. Pró-Fono. 2009; 21(3):249-54.

8. Gschwendtner A, Netzer T, Mairinger B, Mairinger $\mathrm{T}$. What do students think about telemedicine? J Telemed Telecare. 1997; 3(3):169-71. http://dx.doi. org/10.1258/1357633971931093

9. Reese RJ, Aldarondo F, Anderson CR, Lee SJ, Miller TW, Burton D. Telehealth in clinical supervision: a comparison of supervision formats. J Telemed Telecare. 2009; 15(7):356-61. http:// dx.doi.org/10.1258/jtt.2009.090401

10. Winters JM, Winters JM. Videoconferencing and telehealth technologies can provide a reliable approach to remote assessment and teaching without compromising quality. J Cardiovasc Nurs. 2007; 22(1):51-7.

11. Blasca WQ, Bevilacqua MC. A multimídia como uma nova proposta de ensino da audiologia. Salusvita. 2006; 25(3):113-25.

12. Souza AELN. A multimídia como ferramenta de trabalho na Audiologia Educacional: subsídio ao educador de crianças deficientes auditivas [tese]. Bauru (SP): Hospital de Anomalias Craniofaciais da Universidade de São Paulo; 2003.

13. Blasca WQ, Mantovani DA, Campos PD. Fundamentos da psicoacústica aplicados à audiologia: contribuição de recursos de multimídia no ensino aprendizagem. In: XX Encontro Internacional de
Audiologia: Anais do XX Encontro Internacional de Audiologia; São Paulo; 2005.

14. Krumm M, Ferrari DV. Contemporary telehealth and telemedicine applications in audiology. Audiology Today. 2008; 20(5):36-41.

15. Ferrari DV, Blasca WQ, Bastos BG, Bevilacqua MC, Wen CL. Teleaudiología: definiciones y SUS aplicaciones en Brasil [jornal na internet]. Asociación Argentina de Audiología, Buenos Aires. 2008 maio 31[acesso em 18 de fevereiro de 2009]. Disponível em: http://www.asara.org.ar/sec_audclin_art2.asp 16. Ferrari DV, Blasca WQ, Bevilacqua MC, Wen CL. Audiology telehealth research in Brazil. Audinews. 2008; 8(57):4-5.

17. Wen CL. Modelo de ambulatório virtual (cyber ambulatório) e tutor eletrônico (cyber tutor) para aplicação na interconsulta médica e educação à distância mediada por tecnologia [livre docência]. São Paulo (SP): Universidade de São Paulo; 2003. 18. Buck S. Nine human factors contributing to the user acceptance of telemedicine applications: a cognitive-emotional approach. J Telemed Telecare. 2009; 15(2):55-8. http://dx.doi.org/10.1258/ jtt.2008.008007

19. Huang W, Huang W, Diefes-Dux $H$, Imbrie PK. A preliminary validation of attention, relevance, confidenceandsatisfactionmodel-basedinstructional material motivational survey in a computer-based tutorial setting. Br J Educ Technol. 2006; 37(2):24359. http://10.1111/j.1467-8535.2005.00582.x

20. Macea DD, Rondon S, Chaar LJ, Wen CL. Public health education for young students aided by technology. J Telemed Telecare. 2009; 15(3):159. http://dx.doi.org/10.1258/jtt.2009.003018

21. Paixão MP, Miot HA, Souza PE, Haddad AE, Wen CL. A university extension course in leprosy: telemedicine in the Amazon for primary healthcare. J Telemed Telecare. 2009; 15(2):64-7. http://dx.doi. org/10.1258/jtt.2008.080704

DOI: 10.1590/S1516-18462010005000021

RECEBIDO EM: 27/08/2009

ACEITO EM: 02/12/2009

Endereço para correspondência:

Wanderléia Quinhoeiro Blasca

Faculdade de Odontologia de Bauru -

Departamento de Fonoaudiologia

Al. Dr. Octávio Pinheiro Brisola, 9-75

Bauru - SP

CEP: 17012-900

Caixa-Postal: 73

E-mail: wandablasca@usp.br 